Pacific Journal of Mathematic 


\title{
ON THE LINEAR INDEPENDENCE OF ALGEBRAIC NUMBERS
}

\author{
L. J. MORDELL
}

1. Introduction. Besicovitch [1] has proved by elementary methods involving only the concept of the irreducibility of equations the following:

THEOREM. Let

$$
a_{1}=b_{1} p_{1}, a_{2}=b_{2} p_{2}, \cdots, a_{s}=b_{s} p_{s} \text {, }
$$

where $p_{1}, p_{2}, \cdots, p_{s}$ are different primes, and $b_{1}, b_{2}, \cdots, b_{s}$ are positive integers not divisible by any of these primes. If $x_{1}, x_{2}, \cdots, x_{s}$ are positive real roots of the equations

$$
x^{n_{1}}-a_{1}=0, x^{n_{2}}-a_{2}=0, \cdots, x^{n_{s}}-a_{s}=0,
$$

and $P\left(x_{1}, x_{2}, \cdots, x_{s}\right)$ is a polynomial with rational coefficients of degree less than or equ d to $n_{1}-1$ with respect to $x_{1}$, less than or equal to $n_{2}-1$ with respect $t, x_{2}$, and so on, then $P\left(x_{1} x_{2}, \cdots, x_{s}\right)$ can vanish only if all its coefficier ts vanish.

It $i$ is rather surprising that this has not been proved before, since results of this kind occur as particular cases of a general investigation in the theory of algebraic numbers, and some have been known for many years. We have the well-known general problem:

Prólem. Let $K$ be an algebraic number field, and let $x_{1}, x_{2}, \ldots, x_{s}$ be algebraic numbers of degrees $n_{1}, n_{2}, \ldots, n_{s}$ over $K$. When does the field $K\left(x_{1}, x_{2}, \cdots, x_{s}\right)$ have degree $n_{1} n_{2} \ldots n_{s}$ over $K$ ?

This holds if either the degrees or the discriminants over $K$ of the fields $K\left(x_{1}\right), K\left(x_{2}\right), \ldots, K\left(x_{s}\right)$ are relatively prime in pairs. The first part is a simple consequence of the usual theory of reducibility when $s=2$, and the extension is obvious. The second part for $s=2$ is given as Theorem 87 in Hilbert's report on algebraic number fields, and its proof depends on algebraic number

Received July 8, 1952.

Pacific J. Math. 3 (1953), $625-630$ 
theory. The result for general $s$ follows easily.

We discuss here the special case when $x_{1}, x_{2}, \ldots, x_{s}$ are specified roots of the respective equations

$$
x^{n_{1}}=a_{1}, x^{n_{2}}=a_{2}, \cdots, x^{n_{s}}=a_{s},
$$

where $a_{1}, a_{2}, \cdots, a_{s}$ are numbers in $K$. In the particular case when $n_{1} a_{1}$, $n_{2} a_{2}, \ldots, n_{s} a_{s}$ are relatively prime in pairs, the discriminants of the fields $K\left(x_{1}\right), K\left(x_{2}\right), \cdots, K\left(x_{s}\right)$ are certainly relatively prime in pairs, and the foregoing conclusion holds. We consider two types of more general fields $K$. For the first, $K$ and $x_{1}, x_{2}, \ldots, x_{s}$ are all real. For the second, $K$ includes all the $n_{1}$ th, $n_{2}$ th, $\cdots, n_{s}$ th roots of unity, and then the fields

$$
K\left(x_{1}\right), K\left(x_{2}\right), \cdots, K\left(x_{s}\right)
$$

are the so-called Kummer fields and have been known for many years. The elementary ideas used in their discussion are similar to those employed by Besicovitch. We have now the result really asked for in the problem, but stated as follows:

THеовем. A polynomial $P\left(x_{1}, x_{2}, \cdots, x_{s}\right)$ with coefficients in $K$ and of degrees in $x_{1}, x_{2}, \cdots, x_{s}$, less than $n_{1}, n_{2}, \cdots, n_{s}$, respectively, can vanish only if all its coefficients vanish provided that the algebraic number field $K$ is such that there exists no relation of the form

$$
x_{1}^{\nu_{1}} x_{2}^{\nu_{2}} \cdots x_{s}^{\nu_{s}}=a,
$$

where $a$ is a number in $K$, unless

$$
\nu_{1} \equiv 0\left(\bmod n_{1}\right), \nu_{2} \equiv 0\left(\bmod n_{2}\right), \cdots, \nu_{s} \equiv 0\left(\bmod n_{s}\right)
$$

If $K$ is of the first type, then a particular case, which includes the result of Besicovitch and is equivalent to it, arises when $K$ is the rational number field, the $x$ 's are all real, the $a$ 's are integers, $a_{r}(r=1,2, \cdots, s)$ is exactly divisible by a prime power $p_{r}^{\alpha_{r}}$ (that is, by no higher power of $\left.p\right)$ with $\left(\alpha_{r}, n_{r}\right)=$ 1 , the $p_{r}$ are all different, and $p_{r}$ is prime to $a_{t}$ when $r \neq t$. The condition implied in (2) is satisfied, as follows easily from the lemma below.

When $K$ is of the second type, the theorem is given by Hasse [2], in the equivalent form that $K$ includes all the $n$th roots of unity, where $n$ is the least 
common multiple of $n_{1}, n_{2}, \ldots, n_{s}$. Hasse, however, is also investigating the relation of the Galois group of the field $K\left(x_{1}, x_{2}, \cdots, x_{s}\right)$ to those of $K\left(x_{1}\right)$, $K\left(x_{2}\right)$, and so on, and so his proof is not particularly elementary. In view of all this, an elementary proof of the theorem may be worth while.

2. Lemma. We prove first, for completeness, a well-known result:

Lемма. Let $K$ be an algebraic number-field such that either $K$ is real and the equation $x^{n}-a=0$, where $a$ is in $K$, has a real root, or $K$ contains all the nth roots of unity. Then the equation $x^{n}-a=0$ is reducible in $K$ if and only if $a$ is the Nth power of a number in $K$ for some $N>1$ dividing $n$. When $K$ is of the first type, a real root is the root of an irreducible binomial equation in $K$. When $K$ is of the second type, $x^{n}-a$ factorizes completely into binomial factors $x^{m}-b$ in $K$ and irreducible in $K$.

Proof. Let us suppose that $x^{n}-a=0$ is reducible in $K$. Write it as

$$
x^{n}-a=f(x) g(x),
$$

where

$$
f(x)=x^{m}+b_{1} x^{m-1}+\cdots+b_{m},
$$

the $b$ 's are in $K$, and $f(x)$ is irreducible in $K$. When $K$ is of the first type, we may suppose $x^{\prime}$, a specified real root of $x^{n}-a=0$, is a root of $f(x)=0$. All the roots of $f(x)=0$ are roots of $x^{n}-a=0$, and so they have the form $\epsilon^{\prime} x^{\prime}$, where $\epsilon^{\prime}$ is an $n$th root of unity and $x^{\prime}$ is any specified root of $x^{n}-a=0$, but the specified real root when $K$ is of the first type. From the product of the roots of $f(x)=0$,

$$
x^{\prime m}= \pm \epsilon b_{m}
$$

where $\epsilon$ is an $n$th root of unity. Hence $x^{\prime}$ is also the root of an equation

$$
x^{m}=b,
$$

where $b$ is in $K$ since, for the first type, $\epsilon= \pm$ l. Hence the irreducible equation $f(x)=0$ of degree $m$ must be the same as the binomial equation $x^{m}-b=0$.

Further, the equations $x^{n}-a=0, x^{m}-b=0$ have a common root. Write $d=(m, n), n=d N, m=d M$, where $(N, M)=1$ and $a^{M}=b^{N}$. There exist rational integers $u, v$ such that $u M+v N=1$. Then 


$$
a=a^{u M+v N}=\left(b^{u} a^{v}\right)^{N},
$$

where $N \mid n$. Conversely if $a=A^{N}$, where $A$ is in $K$ and $N \mid n$, the equation $x^{n}-a=0$ is obviously reducible in $K$.

This proves the lemma.

3. Proof of theorem. The ideas involved are not essentially different from those of Besicovitch. The given conditions imply that the theorem holds for $s=1$. It will be proved by induction on $s$, and so it may be assumed that no such relation as $P=0$ holds for $s$ or fewer roots of equations satisfying the given conditions. We then prove it for $s+1$ roots. Suppose a relation such as

$$
P\left(x_{1}, x_{2}, \ldots, x_{s+1}\right)=0
$$

holds, so that $x_{1}$ is a root of the equation, supposed irreducible in $K$,

$$
P_{0} x^{r}+P_{1} x^{r-1}+\cdots+P_{r}=0
$$

where $P_{0}, P_{1}, \ldots, P_{r}$ are polynomials with coefficients in $K$, and of degrees in $x_{2}, x_{3}, \ldots, x_{s+1}$ respectively less than $n_{2}, n_{3}, \ldots, n_{s+1}$. Since $1 / P_{0}$ can be expressed as a polynomial in $x_{2}, x_{3}, \ldots, x_{s+1}$ with coefficients in $K$, we may take $P_{0}=1$. We write

$$
P_{1}=P_{1}\left(x_{2}\right)=P_{1}\left(x_{2}, x_{3}, \ldots, x_{s+1}\right),
$$

and so on, according to the variable occuring in $P_{1}$ which we wish to emphasize.

Each root of the equation (4) in $x$ can be written as

$$
x=\epsilon^{\prime} x_{1}, \quad \text { where } \epsilon^{\circ n_{1}}=1
$$

Hence from the product of the roots of (4), $x_{1}$ is also a root of an equation

$$
\epsilon x^{r}= \pm P_{r}
$$$$
\text { where } \epsilon^{n_{1}}=1 \text {, }
$$

Also $\epsilon=1$ when the field $K$ is of the first type. Write

$$
X_{1}=\epsilon x_{1}^{r}, \quad \text { and so } \quad X_{1}^{n_{1}}=a_{1}^{r}
$$

Then by the lemma, $X_{1}$ is a root of an equation irreducible in $K$, 


$$
X_{1}^{N_{1}}=A_{1}
$$

where $A_{1}$ is in $K$. Also,

$$
X_{1}= \pm P_{r}=Q=Q\left(x_{2}\right)=Q\left(x_{2}, x_{3}, \cdots, x_{s+1}\right)
$$

say. Hence the relation ( 3 ) is replaced, when the new variable $X_{1}$ is introduced, by the relation ( 5 ) which is in general simpler. The equation

$$
(Q(x))^{N_{1}}-A_{1}=0
$$

has a root $x=x_{2}$; and since $x^{n_{2}}-a_{2}$ is irreducible in the field $K\left(x_{3}, x_{4}, \ldots\right.$, $\left.x_{s+1}\right)$ by the hypothesis for $s$ variables, each root of $x^{n_{2}}-a_{2}=0$, for example, the conjugate $x_{2}^{\prime}$ of $x_{2}$, must be a root of $(6)$; so

$$
Q\left(x_{2}^{\prime}\right)=X_{1}^{\prime},
$$

where $X_{1}^{\prime}$ is one of the conjugates of $X_{1}$ since $X^{N_{1}}-A_{1}$ is irreducible in $K$.

Now $X_{1}=Q\left(x_{2}\right)$ is the root of the equation in $K\left(x_{3}, x_{4}, \ldots, x_{s+1}\right)$,

$$
F=\left(X-Q\left(x_{2}\right)\right)\left(X-Q\left(x_{2}^{\prime}\right)\right) \cdots=0,
$$

where the product is extended to all the conjugates of $x_{2}$. Since all the roots of the equation $F=0$ in $X$ are conjugates of $X_{1}$, and since, by the hypothesis for $s$ variables, $X^{N_{1}}-A_{1}$ is irreducible in $K\left(x_{3}, x_{4}, \cdots, x_{s+1}\right)$, we must have

$$
F=\left(X^{N_{1}}-A_{1}\right)^{M_{1}}
$$

for some integer $M_{1}>0$, and so $n_{2}=M_{1} N_{1}$. Since $N_{1}>1$, on comparing coefficients of $X^{n_{2}-1}$, we obtain

$$
\sum Q\left(x_{2}^{\prime}\right)=0, \sum X_{1}^{\prime}=0
$$

where the sum is extended over all the conjugates of $x_{2}$ and $X_{1}$, respectively. There are of course exactly $M_{1}$ conjugates of $x_{2}$ which give the same $X_{1}$.

Write now

$$
X=Q(x)=B_{0} x^{n_{2}-1}+B_{1} x^{n_{2}-2}+, \cdots,+B_{n_{2}-1},
$$


where $B_{0}=B_{0}\left(x_{3}, x_{4}, \cdots, x_{s+1}\right)$, and consider all the relations obtained by changing $x$ into $x_{2}$ and all its conjugates. By addition, on noting (7), we get $B_{n_{2}-1}=0$. Write now

$$
X_{1} / x_{2}=X_{1}^{\prime} \text {. }
$$

Then by our condition and by our lemma, $X_{1}^{\prime}$ must be the root of an irreducible equation in $K$,

$$
X^{\prime} N^{\prime}=A^{\prime}
$$

and the conditions involved in (2) still hold. Proceeding as before, we get $B_{n_{2}-2}=0$, and so on until $B_{1}=0$. By the theorem for $s$ variables, a relation such as

$$
X_{1} / x_{2}^{n^{2-1}}=B_{0}
$$

is impossible since $X_{1} / x_{2}^{n_{2}-1}$ is the root of an irreducible binomial equation. This finishes the proof.

\section{REFERENCES}

1. Besicovitch, On the linear independence of fractional powers of integers, J. London Math. Soc. 15 (1940), 3-6.

2. Hasse, Klass enkörpertheorie (Mimeographed lectures, Marburg 1932-33), 187- 195.

St John's College,

CAmbridge, England 


\section{PACIFIC JOURNAL OF MATHEMATICS}

\section{EDITORS}

\author{
R. M. BOEINSON \\ University of California \\ Berkeley 4, California \\ E. HewitT \\ University of Washington \\ Seattle 5 , Washington
}

\section{R. P. DILWOR TH}

California Institute of Technology

Pasadena 4, California

E. F. BECKENBACH

University of California

Los Angeles 24, California

\section{ASSOCIATE EDITORS}

$\begin{array}{ll}\text { H. BUSEMANN } & \text { P. R. HALMOS } \\ \text { HERBERT FFDERER } & \text { IIEINZ HOPF } \\ \text { MARSHALL IJALL } & \text { R. D. JAMES }\end{array}$

\author{
BøRGE JESSEN \\ PAUL LÉVY \\ GEORGE PÓLYA
}

\author{
J. J. STOKER \\ E. G. STR AUS \\ KÔSAKU YOSIDA
}

\section{SFONSORS}

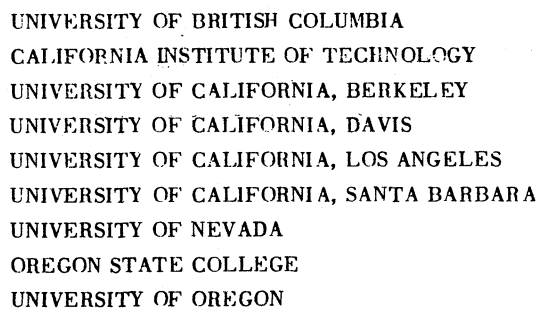

\author{
UNIVERSITY OF SOUTHERN CALIFORNIA \\ STANFORD RESEARCH INSTITUTE \\ STANFORD UNIVERSITY \\ WASHINGTON STATE COLLEGE \\ UNIVERSITY OF WASHINGTON \\ AMERICAN MATHEMATICAL SOCIETY \\ NATIONAL BUREAU OF STANDARDS, \\ INSTITUTE FOR NUMERICAL ANALYSIS
}

Mathematical papers intended for publication in the Pacific Journal of Mathematics should be typewritten (double spaced), and the author should keep a complete copy. Manuscripts may be sent to any of the editors except Robinson, whose term expires with the completion of the present volume; they might also be sent to M.M. Schiffer, Stanford University, Stanford, California, who is succeeding Robinson. All other communications to the editors should be addressed to the managing editor, E. F. Beckenbach, at the address given above.

Authors are entitled to receive 100 free reprints of their published papers and may obtain additional copies at cost.

The Pacific Journal of Mathematics is published quarterly, in March, June, September, and December. The price per volume (4 numbers) is $\$ 8.00$; single issues, $\$ 2.50$. Special price to individual faculty members of supporting institutions and to individual members of the American Mathematical Society: $\$ 4.00$ per volume; single issues, $\$ 1.25$.

Subscriptions, orders for back numbers, and changes of address should be sent to the publishers, University of California Press, Berkeley 4, California.

Printed at Ann Arbor, Michigan. Entered as second class matter at the Post Office, Berkeley, California.

\section{UNIVERSITY OF CALIFORNIA PRESS • BERKELEY AND LOS ANGELES}




\section{Pacific Journal of Mathematics}

\section{Vol. 3, No. 3 \\ May, 1953}

L. Carlitz, Some theorems on generalized Dedekind sums ............ 513

L. Carlitz, The reciprocity theorem for Dedekind sums ............. 523

Edward Richard Fadell, Identifications in singular homology theory..... . . 529

Harley M. Flanders, A method of general linear frames in Riemannian

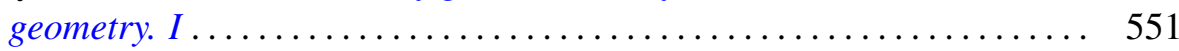

Watson Bryan Fulks, The Neumann problem for the heat equation ........ 567

Paul R. Garabedian, Orthogonal harmonic polynomials.............. 585

R. E. Greenwood and Andrew Mattei Gleason, Distribution of round-off errors for running averages .............................. 605

Arthur Eugene Livingston, The space $H^{p}, 0<p<1$, is not normable ... 613

M. N. Mikhail, On the order of the reciprocal set of a basic set of polynomials .......................................... 617

Louis Joel Mordell, On the linear independence of algebraic numbers . . . . 625

Leo Sario, Alternating method on arbitrary Riemann surfaces .......... 631

Harold Nathaniel Shapiro, Iterates of arithmetic functions and a property of the sequence of primes.............................. 647

H. Shniad, Convexity properties of integral means of analytic functions . . . . 657

Marlow C. Sholander, Plane geometries from convex plates ........... 667 